[Vol. 46,

\title{
254. Ergodic Properties of Piecewise Linear Transformations
}

\author{
By Iekata SHIoKAWA \\ Department of Mathematics, Tokyo Metropolitan University \\ (Comm. by Kinjirô KunUGI, M. J. A., Dec. 12, 1970)
}

1. Introduction. After the work of Rényi [1], ergodic properties of $\beta$-expansions of real numbers have been studied in [2]-[4]. In this paper we generalize these results for a class of expansions, called piecewise linear expansions, which includes $\beta$-expansions as special cases.

Let $\bar{\beta}=\left(\beta_{0}, \beta_{1}, \cdots, \beta_{N}\right), N \geqq 1$, be a $(N+1)$-tuple of positive number such that $0<\theta \equiv \beta_{N}\left(1-\sum_{k=0}^{N-1}\left(1 / \beta_{R}\right)\right) \leqq 1$.

We denote the set of all $(N+1)$-tuples by $V(N+1)$. For each $\bar{\beta} \in V(N+1)$, we define a corresponding function $f(t)$ by

$$
f(t)= \begin{cases}\frac{t}{\beta_{0}}, & 0 \leqq t \leqq 1, \\ f(K)+\frac{t-k}{\beta_{k}}, & k<t \leqq k+1,(k=1,2, \cdots, N+1), \\ 1, & N<t \leqq N+\theta,(k=N), \\ & t>N+\theta .\end{cases}
$$

The function $f(t)$ satisfies the Rényi's conditions [1]. Thus every real number $x$ has the $f$-expansion

$$
x=a_{0}(x)+f\left(a_{1}(x)+f\left(a_{2}(x)+\cdots\right) \cdots\right),
$$

where the digits $a_{n}(x), n=0,1, \cdots$, and the remainders

$$
T^{n} x=f\left(a_{n}(x)+f\left(a_{n+1}(x)+\cdots\right) \cdots\right), \quad n=0,1, \cdots,
$$

are defined by the following recursive relations: $a_{0}(x)=[x], T^{0} x=\{x\}$, $T^{n+1} x=\left\{f^{-1}\left(T^{n} x\right)\right\}, a_{n+1}(x)=\left[f^{-1}\left(T^{n} x\right)\right], n=0,1, \cdots$, where [ $\left.z\right]$ denotes the integral part and $\{z\}$ the fractional part of the real number $z$ and $f^{-1}$ is the inverse function of $f$.

This $f$-expansion is called a piecewise linear expansion induced by $\bar{\beta}$ or simply $\bar{\beta}$-expansion, and the transformation $T x=\left\{f^{-1}(x)\right\}, 0 \leqq x$ $<1$, is called a piecewise linear transformation induced by $\bar{\beta}$. By definition, $T$ is a many to one transformation of $[0,1)$ onto itself and nonsingular with respect to the Lebesgue measure $m$.

For the number 1 , we define, especially, $a_{0}(1)=0$ and $T^{0} 1=1$. Then $\bar{\beta} \in V(N+1)$ is said to be periodic if the $\bar{\beta}$-expansion of 1 has a recurrent tail, and rational if the $\bar{\beta}$-expansion of 1 has a zero tail. The order of $a$ rational $\bar{\beta}$ is the minimum integer $r$ such that $a_{n}(1)=0$ for all $n>r+1$.

2. Invariant measures. Lemma 1. Let $T$ be a piecewise linear transformation induced by $\bar{\beta} \in V(N+1)$ and $\mu$ a finite measure equivalent to the Lebesgue measure $m$. Then $\mu$ is T-invariant if and only if 


$$
h(x)=\sum_{k=0}^{N} f^{\prime}(k+x) h(f(k+x)) d x, \text { a.e. }
$$

where $h(x)$ is the Radon-Nikodym derivative of $\mu$.

Proof. For any $t \in[0,1)$, we have

$$
\mu\left(T^{-1}[0,1)\right)=\int_{0}^{t} \sum_{k=0}^{N} f^{\prime}(k+x) h(f(k+x)) d x .
$$

The lemma is an immediate conclusion of this fact.

For any $\bar{\beta} \in V(N+1)$, we define a function

$$
h(x)=\sum_{n=0}^{\infty} \frac{C_{n}(x)}{\beta_{a_{0}(1)} \beta_{a_{1}(1)} \cdots \beta_{a_{n}(1)}},
$$

where $\beta_{a_{0}(1)}=$ and $C_{n}(x)$ is the characteristic function of the interval $\left[0, T^{n} 1\right)$.

Theorem 1. Let $T$ be a piecewise linear transformation induced by $\bar{\beta}$ and put $\mu(A)=\int_{A} h(x) d x$ for any measurable set $A$. Then $\mu$ is finite T-invariant measure equivalent to the Lebesgue measure.

Proof. First we prove that

$$
\sum_{k=0}^{N} f^{\prime}(k+x) C_{n}(f(k+x))=f\left(a_{n+1}(1)\right)+\frac{C_{n+1}(x)}{\beta_{a_{n+1}(1)}}
$$

If $f(x)>T^{n} 1$, then (1) is trivial. Thus it suffices to prove (1) when there exists an integer $k$ such that $f(k+x)<T^{n} 1$. There are two possibilities: (i) there exists $k$ such that $f(k+x)<T^{n} x<f(k+x)$, (ii) there exists $k$ such that $f(k+1) \leqq T^{n} 1 \leqq f(k+1+x)$. In the case (i) $a_{n+1}(1)=k, C_{n+1}(x)$ $=1$, and in the case (ii) $a_{n+1}(1)=k+1, C_{n+1}(X)=0$. As a result, we get (1). Furthermore, by the piecewise linearity of $f$, we have

$$
1=\sum_{n=0}^{\infty} \frac{f\left(a_{n+1}(1)\right)}{\beta_{a_{0}(1)} \beta_{a_{1}(1)} \cdots \beta_{a_{n}(1)}} .
$$

Therefore, we have

$$
\begin{aligned}
& \sum_{k=0}^{N} f^{\prime}(k+x) h(f(k+x)) \\
= & \sum_{n=0}^{\infty} \frac{1}{\beta_{a_{0}(1)} \beta_{a_{1}(1)} \cdots \beta_{a_{n}(1)}} \sum_{k=0}^{N} f^{\prime}(k+x) C_{n}(f(k+x)) \\
= & \sum_{n=0}^{\infty} \frac{C_{n+1}(x)}{\beta_{a_{0}(1)} \beta_{a_{1}(1)} \cdots \beta_{a_{n+1}(1)}}+\sum_{n=0}^{\infty} \frac{f\left(a_{n+1}(1)\right)}{\beta_{a_{0}(1)} \beta_{a_{1}(1)} \cdots \beta_{a_{n}(1)}} \\
= & h(x) \quad \text { (by (2)). }
\end{aligned}
$$

this and Lemma 1 imply the theorem.

Corollary 1. $h(x)$ is a decreasing jump function which satisfies $1=h(1) \leqq h(x) \leqq h(0)<\infty$, a.e.

Corollary 2. $h(x)$ is a step function with a finite number of steps if and only if $\bar{\beta}$ is periodic. Especially $h(x)=1$ if and only if $\bar{\beta}$ is rational of order 0 .

In what follows we shall investigate the transformation $T$ with the normalized invariant measure $p(\cdot)=\mu(\cdot) / \mu([0,1))$. 
3. Exactness, A measure preserving transformation $T$ on a Lebesgue space $(X, \boldsymbol{B}, P)$ is said to be exact if $\bigcap_{n=0}^{\infty} T^{-n} \boldsymbol{B}=\{X, \emptyset\}$.

Rohlin's criterion [4]. Let $\boldsymbol{U}$ be a countable system of sets of positive measure on $X$ such that the finite unions of pairwise disjoint sets $A \in \boldsymbol{U}$ form an ensemble everywhere dense in $\boldsymbol{B}$. If there exists a positive integer-valued function $n(A), A \in \boldsymbol{U}$, and a positive number $q$ such that $P\left(T^{n(A)} A\right)=1, A \in U$, and

$$
P\left(T^{n(A)} E\right) \leqq q \frac{P(E)}{P(A)},
$$

for all measurable set $E \subset A$ with measurable image $T^{n(A)} E$, then $T$ is exact.

Theorem 2. Every piecewise linear transformation is exact.

Proof. The proof is based on the Rohlin's criterion. Let $\bar{\beta} \in V(N+1)$, be given arbitrary and let us denote by $\xi$ a partition of $(0,1)$ into subintervals generated by the points $f(k), k=1,2, \cdots, N$. We set $\boldsymbol{U}_{n}=\left\{A \in T^{-(n-1)} \xi ; T A \in T^{-(n-2)}\right\}, n=1,2, \cdots, \boldsymbol{U}=\bigcup_{n=1}^{\infty} \boldsymbol{U}_{n}$ and $n(A)$ $=n$ if $A \in U_{n}$. Then, the density and the relation $P\left(T^{n(A)} A\right)=1, A \in U$, are obviously satisfied. We must prove that there exists a constant $q=q(\bar{\beta})$ satisfying the inequality (4). For any $A \in U$, there exists a sequence of digits $\left(a_{1}(A), \cdots, a_{n}(A)\right)$ which is admissible in the $\bar{\beta}$ expansion such that $A=\left(a_{1}(x)=a_{1}(A), \cdots, a_{n}(x)=a_{n}(A)\right)$. Since $T$ is picewise linear, we have $m\left(T^{n(A)} E\right)=\beta_{a_{1}(A)} \cdots \beta_{a_{n}(A)} m(E)=m(E) / m(A)$, for any $E \in B$ in $A$. By this relation and Corollary 1, we obtain $P\left(T^{n(A)} E\right) \leqq h(0)^{2} \mu([0,1))(P(E) / P(A))$. Thus we may set $q=h(0)^{2} \mu([0,1))$.

4. Markov properties. Let $x=\left(a_{1}(x), a_{2}(x), \ldots\right)$ be a $\bar{\beta}$-expansion of a real number $x, 0<x<1$, then $T x=\left(a_{2}(x), a_{3}(x), \ldots\right)$, that is, $T$ is a shift transformation of the stochastic process $\left(a_{1}(x), a_{2}(x), \ldots\right), 0<x<1$, with a finite number of states. Since $P$ is $T$-invariant the process is stationary.

Theorem 3. Let $\bar{\beta}$ be rational of order $r$, then $T$ is a stationary $r$-ple Markov chain. $\quad r=0$ implies the independency of the process.

Lemma 2. Let $\bar{\beta}$ be rational of order $r$ and let $n$ be any nonnegative integer. Then for any sequence of digits $\left(c_{1}, c_{2}, \ldots, c_{n+r}\right)$ which is admissible in the $\bar{\beta}$-expansion, we have

(5) $\quad m\left(\left(c_{n+1}, c_{n+2}, \cdots, c_{n+r}\right)\right)=\beta_{c_{1}} \beta_{c_{2}} \cdots \beta_{c_{n}} m\left(\left(c_{1}, c_{2}, \cdots, c_{n+r}\right)\right)$

where $\left(c_{1}, c_{2}, \cdots, c_{k}\right)=\left(a_{1}(x)=c_{1}, a_{2}(x)=c_{2}, \cdots, a_{k}(x)=c_{k}\right)$.

Proof. If $n=0$, then the relation (5) is trivial. Let $n \geqq 1$. We suppose that (5) holds for $n-1$. Then we have $m\left(\left(c_{2}, c_{3}, \cdots, c_{n+r}\right)\right)=\beta_{c_{2}} \beta_{c_{3}} \cdots \beta_{c_{n}} m\left(\left(c_{n+1}, c_{n+2}, \cdots, c_{n+r}\right)\right)$.

Therefore, we must prove

$$
m\left(\left(c_{2}, c_{3}, \cdots, c_{n+r}\right)\right)=\beta_{c_{1}} m\left(\left(c_{1}, c_{2}, \cdots, c_{n+r}\right)\right)
$$

for any admissible sequence $\left(c_{1}, c_{2}, \cdots, c_{n+r}\right)$. Here (6) holds obviously 
for $c_{1}=0,1, \cdots, N-1$. Thus it remains to show that (6) holds for $c_{1}=N$. To do this it suffices to prove

$$
\left(c_{2}, c_{3}, \cdots, c_{n+r}\right) \subset[0, T 1) .
$$

Since $\bar{\beta}$ is rational of order $r, T 1$ is an endpoint of an interval $\left(c_{1}^{\prime}, c_{2}^{\prime}\right.$, $\cdots, c_{k}^{\prime}$ ) of length $k \geqq r$. So we have

$$
\left(c_{2}, c_{3}, \cdots, c_{n+r}\right) \subset[0, T 1) \quad \text { or } \quad\left(c_{2}, c_{3}, \cdots, c_{n+r}\right) \subset[T 1,1) .
$$

But the last relation contradicts the admissibility of the sequence $\left(N, c_{2}, \cdots, c_{n+1}\right)$. Thus we have the relation (7). By induction the lemma is proved.

Proof of Theorem 1. By Lemma 2, we have

$$
\begin{aligned}
m\left(a_{n+r+1}(x)\right. & \left.=c_{n+r+1} ; a_{1}(x)=c_{1}, \cdots, a_{n+r}(x)=c_{n+r}\right) \\
& =\frac{m\left(\left(c_{n+1}, c_{n+2}, \cdots, c_{n+r+1}\right)\right)}{m\left(\left(c_{n+1}, c_{n+2}, \cdots, c_{n+r}\right)\right)}=Q\left(c_{n+1}, \cdots, c_{n+r+1}\right)
\end{aligned}
$$

where $Q$ is a constant which depends only on the admissible sequence $\left(c_{n+1}, c_{n+2}, \cdots, c_{n+r+1}\right)$. Since $\bar{\beta}$ is rational of order $r, h(x)$ is constant on every interval $\left(c_{1}, c_{2}, \cdots, c_{k}\right)$ of length $k \geqq r$. Then, we have

$$
P\left(a_{n+r+1}(x)=c_{n+r+1} ; a_{1}(x)=c_{1}, \cdots, a_{n+r}(x)=c_{n+r}\right)=Q\left(c_{n+1}, \cdots, c_{n+r+1}\right)
$$

for any admissible sequence $\left(c_{1}, c_{2}, \cdots c_{n+r+1}\right)$.

\section{References}

[1] Rényi, A.: Representations for real numbers and their ergodic properties. Acta Math. Acad. Sci. Hung., 8, 477-493 (1957).

[2] Parry, W.: On the $\beta$-expansions of real numbers. Acta Math. Sci. Hung., 11, 401-416 (1960).

[ 3 ] Cigler, J.: Ziffenverteilung in $\vartheta$-adischen Büchen. Math. Zeit., 75, 8-13 (1961).

[ 4 ] Rohlin, V. A.: Exact endomorphisms of a Lebesgue space. Izv Akad. Nauk SSSR, 25, 499-530 (1961). Amer. Math. Soc. Transl., 39 (2), 1-36 (1964). 